\title{
Rigorous conditions for food-web intervality in high-dimensional trophic niche spaces
}

Åke Brännström (ake.brannstrom@math.umu.se)

Linus Carlsson (linus.carlsson@math.umu.se)

Axel G. Rossberg (axel@rossberg.net)

\section{Approved by}

Ulf Dieckmann

Program Leader, EEP

July 2011 


\title{
Rigorous conditions for food-web intervality in high-dimensional trophic niche spaces
}

\author{
Åke Brännström, Linus Carlsson, and Axel G. Rossberg
}

January 2010

\begin{abstract}
Food webs represent trophic (feeding) interactions in ecosystems. Since the late 1970s, it has been recognized that food-webs have a surprisingly close relationship to interval graphs. One interpretation of food-web intervality is that trophic niche space is low-dimensional, meaning that the trophic character of a species can be expressed by a single or at most a few quantitative traits. In a companion paper we demonstrated, by simulating a minimal food-web model, that food webs are also expected to be interval when niche-space is high-dimensional. Here we characterize the fundamental mechanisms underlying this phenomenon by proving a set of rigorous conditions for food-web intervality in high-dimensional niche spaces. Our results apply to a large class of food-web models, including the special case previously studied numerically.
\end{abstract}

\section{Introduction}

The world's ecosystems supply a range of provisioning, regulating, supporting, and cultural services on which humans depend (Millennium Ecosystem Assessment, 2005). At the heart of an ecosystem is an intricate and multifactorial network of species interactions. Food webs reflect the flow of energy within these networks that emerge from predation and consumption (Pimm, 1982). In their most abstract form, food webs can be described by unweighted directed graphs, where vertices represent species and edges indicate energy flows between vertices. Because of their fundamental role for ecosystems, food webs have been studied with mounting interest since the 1950s in order to elucidate general structural patterns and to better understand their implications for demographic and evolutionary dynamics (Bersier, 2007).

One of the more surprising findings to date is that food webs are closely related to interval graphs as defined by Benzer (1959), a phenomenon now known as food-web intervality. Cohen (1977) was the first to point out this striking regularity in empirically measured food webs. Starting with a directed graph representing a food web, he defined an associated 'niche overlap graph' where each species forms a node, and two nodes are connected if the species have at least one resource (prey species) in common. Cohen $(1977,1978)$ showed that empirical niche overlap graphs are interval much more often than would be expected by chance. Since Cohen (1977)'s seminal discovery, many other characterizations of food-web intervality have been proposed (Sugihara, 1984; 
Stouffer et al, 2006). Among them is the consecutive ones property (COP). A matrix of ones and zeros is said to have the COP along the columns if there is an ordering of its rows such that the ones appear consecutively along the columns. For the adjacency matrix of a food webs, this corresponds to an ordering of resources such that the diet of each consumer (predator species) forms a contiguous block in this ordering.

A common explanation for food-web intervality assumes that vulnerability of each species to predation can be described by a point on a line, that the trophic (feeding) niche of each consumer is given by an interval on that line, and that the diet of each consumer is then given by all species with vulnerability traits in its trophic niche. This picture would not only imply that the niche overlap graph is interval, but also that the food web has the COP. For this reason, many authors have argued that the trophic 'niche space' is one-dimensional (e.g., Cohen, 1977; Williams and Martinez, 2000; Stouffer et al, 2006; Mouillot et al, 2008; Allesina and Pascual, 2009). This line of though is so influential that it has become the basis of one of the most widely-used food-web models, the niche model by Williams and Martinez (2000). In this model, vulnerability traits are independent and identically distributed random numbers from the unit interval $[0,1]$, and feeding intervals are placed randomly under the constraint that they fit into $[0,1]$ and their center value is smaller than the consumer's vulnerability trait.

In a companion paper (Rossberg et al, 2010a), we introduced a food-web model which draws on similar ideas, but where species are not necessarily characterized by a single real value. Instead, species are characterized by 'vulnerability traits' and 'foraging traits' which are vectors in a $D$-dimensional trait space. The main elements of the model are illustrated in Figure 1. In a natural generalization of intervals, consumers feed on all species with vulnerability traits within a $D$-dimensional disc. The centre of this disc is given by the vector of foraging traits. As in the niche model, vulnerability traits are randomly assigned, but in stark contrast they are not independently sampled. Instead, they derive from a branching mean-reverting random walk that mimics the evolutionary process from which ecological communities emerge (Felsenstein, 1988; Blomberg et al, 2003a), where branching events correspond to speciations. This induces phylogenetic correlations between vulnerability traits. Foraging traits are determined in ways that model fast adaptation, with the simplest choice being the assumption that each consumer is specialized to feed on one specific resource, but also consumes other resources that fall within its trophic niche. Using this model, Rossberg et al (2010a) demonstrated in simulations that food webs do not only have the COP when the niche-space is one-dimensional, i.e., $D=1$, but that the probability of food-webs having the COP (and therefore being interval) also tends to 1 as the dimension $D \rightarrow \infty$.

Moving away from simulations of specific models, the aim of this paper is to derive mathematically rigorous conditions for food-web intervality in higherdimensional trophic niche spaces. In Sect. 2 we define a generic family of food web models and required associated terms. Based on these definitions, we state and prove a theorem in Sect. 3 characterizing conditions under which a food webs assume the COP as $D \rightarrow \infty$. One of the conditions is that consumers are 'focused', meaning that the foraging traits of a consumer are exactly equal to the vulnerability traits of a resource. In Sect. 4 we partly relax this assumption, requiring only that the foraging traits stay closer to the nearest vulnerability 

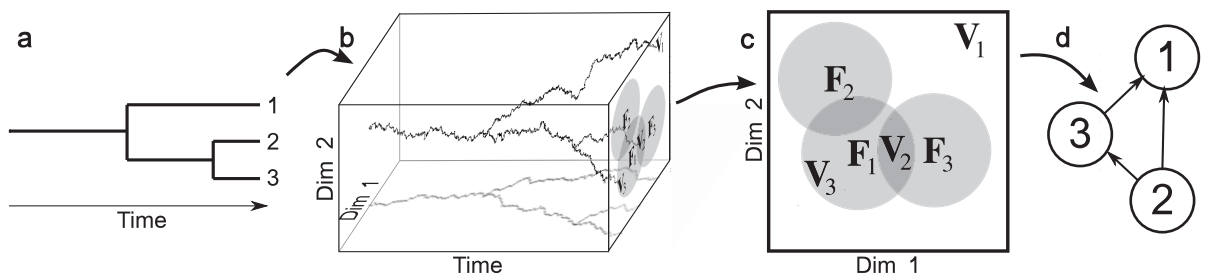

Figure 1: A schematic illustration of the food-web model considered in this paper. Each species $s_{i}$ is characterized by a vulnerability trait vector $\vec{V}_{i}$ and a foraging trait vector $\vec{F}_{j}$ (here vectors in $\mathbb{R}^{2}$ ). a) Species descend from an $a$ priori prescribed phylogenetic tree, with $T_{i j}$ being the time since separation of the lineages $i$ and $j$. b) The vulnerability trait vectors are determined by a mean-reverting random walk (Ornstein-Uhlenbeck process) which branches at the times given by the phylogenetic tree. c) Two species are linked as consumer and resource if the vulnerability trait vector of the resource is closer to the foraging trait vector of the consumer than a prescribed niche width. d) The resultant food web. Vertices represent species and edges indicate the direction of energy flows.

trait than a distance on the order of $\sqrt{D}$. We show that this bound on distance is sharp in the sense that, at larger distances, other rules have to be followed for the placement of foraging trait to ensure intervality as $D \rightarrow \infty$. Two plausible rules are discussed, with one leading to intervality and the other not. Finally, we conclude with a discussion of our main results and the two major underlying ecological assumptions: fast consumer adaptation and focused foraging traits.

\section{Preliminaries}

We first give mathematical definitions for key ecological terms. These will later allow us to state and prove our main theorem.

Definition 1. A food web $(S, L)$ consists of a finite set of species $S$ and a set of directed links $L \subset S \times S$. We call a species $c \in S$ a consumer if $(r, c) \in L$ for some $r \in S$. Similarly, we call a species $r \in S$ a resource if $(r, c) \in L$ for some $c \in S$.

Stated plainly, a food web is a directed graph and a species is a consumer (resource) if there is an associated incoming (outgoing) link. These links represent energy flows in the system. Note that a species can be both a consumer and a resource. We now associate each species with trophic traits and specify how these induce a food web, thus giving precise meaning to the concept of niche space.

Definition 2. Let $S$ be a finite set of species and $D$ be a positive integer. Associate with each species $s \in S$ a trait vector $\left(\vec{V}_{s}, \vec{F}_{s}, w_{s}\right) \in \mathbb{R}^{D} \times \mathbb{R}^{D} \times \mathbb{R}_{+}$ consisting of vulnerability traits $\vec{V}_{s} \in \mathbb{R}^{D}$, foraging traits $\vec{F}_{s} \in \mathbb{R}^{D}$, and a niche width $w_{s}>0$. By the induced food web we mean the food web $(S, L)$ with the set of directed links given by

$$
L=\left\{(r, c):\left|\vec{V}_{r}-\vec{F}_{c}\right|^{2} \leq D w_{c}^{2}\right\} .
$$


We will often use the adjacency matrix $A(L)$ of $L$ defined by the $D \times D$ matrix $A(L)=\left(a_{i j}\right)$, where $a_{i j}=1$ if $\left(s_{i}, s_{j}\right) \in L$ and $a_{i j}=0$ otherwise.

An ecological interpretation of this definition is that species need to have foraging traits sufficiently close to other species' vulnerability traits in order to be effective consumers. Later on we will take the limit of an infinite-dimensional niche space, $D \rightarrow \infty$, and it is for this reason that we scale the niche width in Eq. (1) with $D$.

We now define the consecutive-ones property which is a strong characterization of describe food-web intervality in the sense that it implies all other commonly used descriptors (see Fig. 2b of Rossberg et al, 2010a).

Definition 3. A binary matrix, i.e., a matrix with entries belonging to the set $\{0,1\}$, is said to have the consecutive ones property (COP) if there exists a permutation of the rows so that all 1's along the columns are consecutive. A food web is said to have the COP if its adjacency matrix has the COP.

A simple way to model fast consumer adaptation is to assume that each consumer is focused on a specific resource:

Definition 4. For any finite set of species with associated trait vectors as in Definition 2, call a consumer $c \in S$ focused if there is a some focal resource $r \in S$ such that $\vec{F}_{c}=\vec{V}_{r}$

Definition 5. A species $c \in S$ is called basal if it does not consume any other species; this implies that the entries in the column corresponding to $c$ in the matrix $A(L)$ are all zero.

We consider a class of food webs where all species have vulnerability traits given by a branching Ornstein-Uhlenbeck process, a branching random walk with a drift towards the origin. The branching events are interpreted as speciations in a phylogenetic tree. For our argument, the times since the last common ancestor for any species pair can be chosen arbitrarily, subject to a consistency requirement for phylogenetic distance matrices:

Definition 6. Let $T$ be an $m \times m$ symmetric real matrix with non-negative entries. We say that $T$ is a phylogenetic distance matrix of order $m$ if for all $i$ we have $T_{i i}=0$ and for any three distinct integers $1 \leq i, j, k \leq m$ we have $T_{i j} \leq \max \left\{T_{i k}, T_{j k}\right\}$.

In the mathematical literature such a matrix is called an ultrametric.

From Def. 6 we derive the following two lemmas which will play a central role in proving conditions for food-web intervality in Sect. 3 and Sect. 4. Although the lemmas may at first sight appear technical, the idea they encapsulate is surprisingly simple as illustrated in Fig. 2.

Lemma 1. Given any phylogenetic distance matrix $T$ of order $m$ we can find a permutation $\tau$ of the integers $\{1, \ldots, m\}$ such that for any integers $1 \leq i \leq j \leq$ $k \leq m$ the matrix $T^{\prime}$ defined by $T_{i j}^{\prime}=T_{\tau(i) \tau(j)}$ satisfies $T_{i j}^{\prime} \leq T_{i k}^{\prime}$.

Proof. It is known that every phylogenetic distance matrix corresponds to a rooted phylogenetic tree with equidistant edge weightings (see e.g. Semple and Steel, 2003, Theorem 7.2.5). A pre-order traversal of the rooted phylogenetic tree determined by $T$, starting from the root, induces an ordering of vertices whose restriction to the leaves is a permutation with the desired property. 
a

$$
T=\left(\begin{array}{llll}
0 & 5 & 5 & 5 \\
5 & 0 & 3 & 1 \\
5 & 3 & 0 & 3 \\
5 & 1 & 3 & 0
\end{array}\right)
$$

b

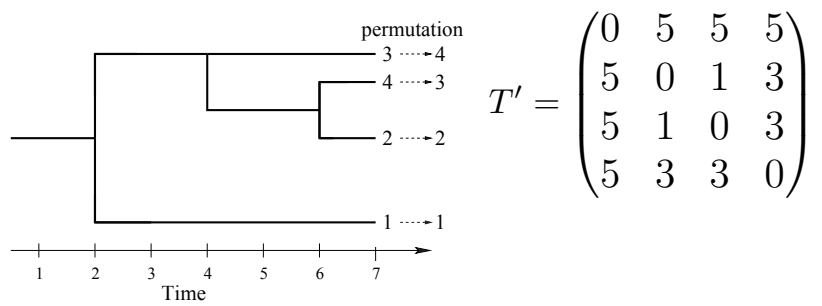

Figure 2: a) A phylogenetic distance matrix as in Def. 6. As an example of how the matrix should be interpreted, the phylogenetic distance between species 2 and 4 is 1 , since $T_{24}=T_{42}=1$. b) One possible associated rooted phylogenetic tree with equidistant edge weightings. A pre-order traversal of the tree yields the permutation $\{1,2,4,3\}$. c) Relabeling the species according to the permutation gives a new phylogenetic distance matrix $T^{\prime}$ where the elements in each row increase to the left and to the right from the diagonal (c.f. Lemma 1 and Lemma 2).

In what follows we assume without loss of generality that all phylogenetic distance matrices have the ordering prescribed in Lemma 1. (That is, they are in an order in which they would be when the phylogenetic tree is drawn in the conventional form as a planar graph.)

Lemma 2. Let $T$ be a phylogenetic distance matrix of order $m$. Then, for any integer $1 \leq c \leq m$ and any triple of integers $1 \leq i<j<k \leq m$, we have $T_{c j} \leq \max \left\{T_{c i}, T_{c k}\right\}$.

Proof. Let $c, i, j, k$ be as in the statement of the lemma. Since $T$ is an ultrametric and symmetric we have

$$
T_{c j} \leq \max \left\{T_{c i}, T_{j i}\right\} .
$$

Now, from symmetry and Lemma 1 ,

$$
T_{j i}=T_{i j} \leq T_{i k} \leq \max \left\{T_{i c}, T_{k c}\right\}=\max \left\{T_{c i}, T_{c k}\right\}
$$

Combining relations (2) and (3) we have

$$
T_{c j} \leq \max \left\{T_{c i}, T_{c k}\right\}
$$

which concludes the proof.

We can now define the branching Ornstein-Uhlenbeck process from which we will sample vulnerability traits.

Definition 7. Let $T$ be a phylogenetic distance matrix of order $m$ and let $\vec{W}_{i}$, $i=1, \ldots, m$ be D-dimensional Wiener processes such that $\vec{W}_{i}$ and $\vec{W}_{j}$ with $i \neq j$ have identical increments for $t<-T_{i j}$ and independent increments otherwise. From these we form $m$ Ornstein-Uhlenbeck processes in $\mathbb{R}^{D}$ as the solutions to the stochastic differential equations,

$$
d \vec{V}_{i}(t)=-r \vec{V}_{i}(t) d t+\sigma d \vec{W}_{i},
$$

with initial conditions chosen so that all $\vec{V}_{i}$ coincide for $t<-\max _{i, j} T_{i, j}$. By a sample from a D-dimensional branching Ornstein-Uhlenbeck process associated with $T$ we mean a sample of the random vectors $\vec{V}_{i}, i=1, \ldots, m$, at $t=0$. 
The definition implies that the vulnerability trait vectors of two species $V_{i}$ and $V_{j}$ are identical for $t<-T_{i j}$. A branching event then occurs at time $t=T_{i j}$, after which the two species vulnerability trait vectors evolve independently. This is illustrated in Fig. 1b and is intended as a simple model of speciation.

Lemma 3. For a branching Ornstein-Uhlenbeck process as defined above and integers $1 \leq i, j \leq n$, the squared difference $\left|\vec{V}_{i}(t)-\vec{V}_{j}(t)\right|^{2}$ is distributed as $v\left(T_{i j}\right) \chi_{D}^{2}$, where $\chi_{D}^{2}$ is chi-squared distributed with $D$ degrees of freedom and the function $v: \mathbb{R}_{+} \mapsto \mathbb{R}_{+}$is defined by

$$
v(t):=\frac{\sigma^{2}}{r}[1-\exp (-2 r t)]
$$

Proof. Let two integers $1 \leq i, j \leq n$ be given. If $i=j$, the assertion is trivially true as $T_{i i}=0$ for any $i$. Thus, assume that $i \neq j$ and define $\vec{X}(t)=\vec{V}_{i}(t)-\vec{V}_{j}(t)$. For $t \leq-T_{i j}, \vec{X}(t)=0$. For $t>-T_{i j}$, it follows from Ito's formula and (4) that

$$
d \vec{X}(t)=-r \vec{X}(t) d t+\sqrt{2} \sigma d \vec{W},
$$

where $\vec{W}$ is a $D$-dimensional Wiener process. By Eq. (6), all $D$ components of $\vec{X}(t)$ are independent Ornstein-Uhlenbeck processes. From $\vec{X}\left(-T_{i j}\right)=0$ and standard results on one-dimensional Ornstein-Uhlenbeck processes (see e.g. Gardiner, 2009, pp. 105-106), it follows that $\vec{X}(t)$ is normally distributed for $t>-T_{i j}$ and that the variance of each respective component is $v\left(T_{i j}-t\right)$ where $v(t)$ is defined by $(5)$. Hence, $\vec{X}(0)$ is a vector of independent normally distributed random variables, each with variance $v\left(T_{i j}\right)$. The assertion that $\left|\vec{V}_{i}(t)-\vec{V}_{j}(t)\right|^{2}=|\vec{X}(0)|^{2}=v\left(T_{i j}\right) \chi_{D}^{2}$ thus follows directly from the definition of the $\chi^{2}$-distribution.

\section{Food-web intervality with focused consumers}

We now state our main result:

Theorem 1. Let $S=\left\{s_{1}, \ldots, s_{m}\right\}$ be a finite set of species and $T$ a phylogenetic distance matrix of order $m$. For each $D>0$ we associate with each species $s_{i}$ a trait vector $\left(\vec{V}_{i}^{D}, \vec{F}_{i}^{D}, w_{i}\right)$ where the $\vec{V}_{i}^{D}$ are sampled from a D-dimensional branching Ornstein-Uhlenbeck process and the niche widths $w_{i}>0, i=1, \ldots, m$, are independent of $D$ and satisfy $\left|w_{i}^{2}-v\left(T_{j k}\right)\right|>\epsilon$ for all $1 \leq i, j, k \leq m$ and some $\epsilon>0$. If all consumers are focused, then the probability that the induced food web has the COP (and therefore is interval) tends to 1 as $D \rightarrow \infty$.

Remark 1. Alternatively, we could assume that $w_{i}^{2} \neq v\left(T_{j k}\right)$ for any $1 \leq$ $i, j, k \leq m$, as this is equivalent to the existence of an $\epsilon>0$ such that $\mid w_{i}^{2}-$ $v\left(T_{j k}\right) \mid>\epsilon$ for all $1 \leq i, j, k \leq m$. Even though the degenerate case where $w_{i}^{2}=$ $v\left(T_{j k}\right)$ for some $i, j, k$ is not likely to occur in practice, food-web intervality will be less pronounced for any finite dimension $D$ when the squared niche widths $w_{i}^{2}$ are close to $v\left(T_{j k}\right)$. Vulnerability trait vectors will then be close to the boundary of being included in a consumer's diet and may fall in or out by random chance.

Remark 2. For establishing the COP property of $A(L)$ we only need to consider species that consume more than one resource, as otherwise there are less than two ones in the column of consideration. (For basal species there are just zeros). 
Remark 3. In the proof of Theorem 1, and in the remainder of this manuscript, we suppress the dependence of foraging traits, vulnerability traits, and related quantities on the dimension $D$.

Theorem 1. We prove the theorem in two steps. First we show that for any $D>0$ the induced food web has the COP if for any integers $1 \leq i, j \leq m$ we have

$$
-\frac{\epsilon}{2}<\frac{\left|\vec{V}_{i}-\vec{V}_{j}\right|^{2}}{D}-v\left(T_{i j}\right)<\frac{\epsilon}{2} .
$$

Second, we show that the probability that (7) holds for all $1 \leq i, j \leq m$ simultaneously tends to 1 as $D \rightarrow \infty$. For notational simplicity we will throughout the proof identify a species $s_{i}$ with its index $i$.

Step 1. Assume that the food web does not have the consecutive ones property when species are ordered as in the phylogenetic distance matrix. We will show that this leads to a contradiction. That is, we can find a consumer $1 \leq c \leq m$ and species $1 \leq i<j<k \leq m$ such that the consumer $c$ has $i$ and $k$ among its resources, but not $j$. Denote by $r$ the species on which the consumer is focused. Then $\vec{F}_{c}=\vec{V}_{r}$ and,

$$
\left|\vec{V}_{r}-\vec{V}_{i}\right|^{2} \leq D w_{c}^{2},\left|\vec{V}_{r}-\vec{V}_{j}\right|^{2}>D w_{c}^{2} \text {, and }\left|\vec{V}_{r}-\vec{V}_{k}\right|^{2} \leq D w_{c}^{2} .
$$

Let

$$
\epsilon_{r i}=\frac{\left|\vec{V}_{r}-\vec{V}_{i}\right|^{2}}{D}-v\left(T_{r i}\right)
$$

We can now rewrite (8) as

$$
v\left(T_{r i}\right)+\epsilon_{r i} \leq w_{c}^{2}, v\left(T_{r j}\right)+\epsilon_{r j}>w_{c}^{2} \text {, and } v\left(T_{r k}\right)+\epsilon_{r k} \leq w_{c}^{2} .
$$

From Lemma 2 and the fact that $v$ is an increasing function we have $v\left(T_{r j}\right) \leq$ $\max \left\{v\left(T_{r i}\right), v\left(T_{r k}\right)\right\}$. Thus, $v\left(T_{r j}\right) \leq v\left(T_{r i}\right)$ or $v\left(T_{r j}\right) \leq v\left(T_{r k}\right)$. Noting that the two cases are similar, we only show that a contradiction ensues assuming the former inequality. If, instead the latter inequality holds, an analogous argument applies. This gives,

$$
-\frac{\epsilon}{2}<\epsilon_{r i} \leq w_{c}^{2}-v\left(T_{r i}\right) \leq w_{c}^{2}-v\left(T_{r j}\right)<\epsilon_{r j}<\frac{\epsilon}{2},
$$

where we have additionally used (7) and (9). This contradicts the assumption in the theorem that $\left|w_{c}^{2}-v\left(T_{\text {ri }}\right)\right|>\epsilon$.

Step 2. For the this part of the proof we first fix two integers $1 \leq i, j \leq m$ and observe that $D^{-1}\left|\vec{V}_{i}-\vec{V}_{j}\right|^{2}$ is a sample average of $D$ independent and identically distributed random variables. Thus, by the weak law of large numbers the sum converges in probability to its expected value,

$$
\frac{\left|\vec{V}_{i}-\vec{V}_{j}\right|^{2}}{D} \stackrel{\mathrm{P}}{\rightarrow} v\left(T_{i j}\right) \text { as } D \rightarrow \infty
$$

Thus, by definition, the probability that (7) holds for this particular pair of integers tends to 1 as $D \rightarrow \infty$. To see that the probability that (7) holds for all pairs of integers $1 \leq i, j \leq m$ also tends to 1 , we note that we can bound the joint probability of two simultaneous but not necessarily independent events as $\mathrm{P}\left(A_{1} \wedge A_{2}\right) \geq \mathrm{P}\left(A_{1}\right)+\mathrm{P}\left(A_{2}\right)-1 \geq 2 \min \left(\mathrm{P}\left(A_{1}\right), \mathrm{P}\left(A_{2}\right)\right)-1$, and more generally of $n$ simultaneous events as $\mathrm{P}\left(\bigwedge_{i=1}^{n} A_{i}\right) \geq 1-n+n \min _{i=1}^{n} \mathrm{P}\left(A_{i}\right)$. Hence, for any finite $n$ we have that $\mathrm{P}\left(\bigwedge_{i=1}^{n} A_{i}\right) \rightarrow 1$ whenever $\mathrm{P}\left(A_{i}\right) \rightarrow 1$ for all $i$. This completes the proof. 
Remark 4. Using Chebyshev's inequality one can show that the rate of convergence in (10) is at least

$$
\mathrm{P}\left(\left|\frac{\left|\vec{V}_{i}-\vec{V}_{j}\right|^{2}}{D}-v\left(T_{i j}\right)\right| \leq \frac{\varepsilon}{2}\right) \geq 1-\frac{8}{D \varepsilon^{2}}
$$

To see this, consider the complementary event ||$\vec{V}_{i}-\left.\vec{V}_{j}\right|^{2}-D v\left(T_{i j}\right) \mid>D \varepsilon / 2$ and notice that, by Lemma 3, the variance of $\left|\vec{V}_{i}-\vec{V}_{j}\right|^{2}$ is $2 D v\left(T_{i j}\right)^{2} \leq 2 D$.

Remark 5. A central element of the proof is the convergence in (10) of the squared difference of vulnerability vectors to the expected value $v\left(T_{i j}\right)$. We based our argument on the weak law of large numbers, as this immediately leads us to a statement on the satisfiability of (7). It is, however, easy to prove convergence almost surely and thus establish a strong law of large numbers. Indeed, this follows directly from standard theorems, such as Theorem 3 p. 294 of Grimmett and Stirzaker (1992).

We derive the following corollary:

Corollary 1. Theorem 1 also holds when the niche widths $w_{i}$ are independent samples from a continuous random variable taking values in $[0, \infty)$ without any restriction on the proximity to the points $v\left(T_{i j}\right), 1 \leq i, j \leq m$.

Proof. Let $W_{D}=\left(S, L_{D}\right)$ be the induced food web in dimension $D$. If we can show that for any $\epsilon>0$,

$$
\limsup _{D \rightarrow \infty} \mathrm{P}\left(W_{D} \text { violates } \mathrm{COP}\right) \leq \epsilon,
$$

then, since $\epsilon>0$ is arbitrary and probabilities are non-negative, it follows that

$$
\lim _{D \rightarrow \infty} \mathrm{P}\left(W_{D} \text { violates } \mathrm{COP}\right)=0
$$

exists and is equal to 0 , which proves the corollary. To prove the assertion choose $\delta>0$ such that $\mathrm{P}\left(\left|w_{c}^{2}-v\left(T_{i j}\right)\right|<\delta\right.$ for all $\left.1 \leq c, i, j \leq m\right)<\epsilon$. This is possible since the cumulative distribution function of a continuous random variable is continuous. Then,

$$
\begin{aligned}
\mathrm{P}\left(W_{D} \text { violates } \mathrm{COP}\right) & \\
= & \mathrm{P}\left(\left|w_{c}^{2}-v\left(T_{i j}\right)\right|<\delta\right) \mathrm{P}\left(W_{D} \text { violates } \mathrm{COP}|| w_{c}^{2}-v\left(T_{i j}\right) \mid<\delta\right) \\
& +\mathrm{P}\left(\left|w_{c}^{2}-v\left(T_{i j}\right)\right| \geq \delta\right) \mathrm{P}\left(W_{D} \text { violates } \mathrm{COP}|| w_{c}^{2}-v\left(T_{i j}\right) \mid \geq \delta\right) \\
\leq & \epsilon+\mathrm{P}\left(W_{D} \text { violates } \mathrm{COP}|| w_{c}^{2}-v\left(T_{i j}\right) \mid \geq \delta\right),
\end{aligned}
$$

and from Theorem 1 we know that the latter term tends to 0 as $D \rightarrow \infty$.

\section{Food-web intervality with weakly focused con- sumers}

In this section we study food webs in situations where the consumers are weakly focused, i.e., have foraging trait vectors which are close to, but not identical 
with, other species' vulnerability trait vectors. In particular, we show that Theorem 1 is still valid in the weakly focused case under a particular restriction on the location of the foraging trait vectors. The arguments in the proof of this section are more intricate, because a consumer's foraging trait vector can not be substituted for a vulnerability trait vector it is focused on. For this reason we have presented these results separately.

Theorem 2. Let $S=\left\{s_{1}, \ldots, s_{m}\right\}$ be a finite set of species and $T$ a phylogenetic distance matrix of order $m$. For each $D>0$ we associate with each species $s_{i}$ a trait vector $\left(\vec{V}_{i}^{D}, \vec{F}_{i}^{D}, w_{i}\right)$ where the $\vec{V}_{i}^{D}$ are sampled from a D-dimensional branching Ornstein-Uhlenbeck process and the niche widths $w_{i}>0, i=1, \ldots, m$, are independent of $D$ and satisfy $\left|w_{i}^{2}-v\left(T_{j k}\right)\right|>\epsilon$ for some $\epsilon>0$ and all $1 \leq i, j, k \leq m$. If to every consumer $c$ and every dimension $D$ there exist a resource $r_{D}$ such that

$$
\frac{\left|\vec{F}_{c}-\vec{V}_{r_{D}}\right|^{2}}{D} \stackrel{\mathrm{P}}{\rightarrow} 0 \text { as } D \rightarrow \infty
$$

then the probability that the induced food web has the COP (and therefore is interval) tends to 1 as $D \rightarrow \infty$.

Proof. We follow the proof of Theorem 1. We first show that for any $D>0$ the induced food web has the COP if for all integers $1 \leq i \leq m$ and all consumers $c \in S$ we have that

$$
-\frac{\epsilon}{2}<\frac{\left|\vec{F}_{c}-\vec{V}_{i}\right|^{2}}{D}-v\left(T_{r i}\right)<\frac{\epsilon}{2},
$$

where $r$ is defined as the first integer such that

$$
\left|\vec{F}_{c}-\vec{V}_{r}\right|=\min _{i \in S}\left|\vec{F}_{c}-\vec{V}_{i}\right|
$$

Second, we show that the probability that (12) holds for all $1 \leq i \leq m$ and all consumers $c \in S$ simultaneously tends to 1 as $D \rightarrow \infty$. For notational simplicity we will throughout the proof identify a species $s_{i}$ with its index $i$.

Step 1. Assume that the food web does not have the consecutive ones property when species are ordered as in the phylogenetic distance matrix. We will show that this leads to a contradiction. That is, we can find a consumer $1 \leq c \leq m$ and species $1 \leq i<j<k \leq m$ such that the consumer $c$ has $i$ and $k$ among its resources, but not $j$. Let $r$ be denoted as in (13). Then,

$$
\left|\vec{F}_{c}-\vec{V}_{i}\right|^{2} \leq D w_{c}^{2},\left|\vec{F}_{c}-\vec{V}_{j}\right|^{2}>D w_{c}^{2} \text {, and }\left|\vec{F}_{c}-\vec{V}_{k}\right|^{2} \leq D w_{c}^{2} .
$$

Let

$$
\epsilon_{c i}=\frac{\left|\vec{F}_{c}-\vec{V}_{i}\right|^{2}}{D}-v\left(T_{r i}\right) \text { where } 1 \leq c, i \leq m .
$$

We can now rewrite (14) as

$$
v\left(T_{c i}\right)+\epsilon_{c i} \leq w_{c}^{2}, v\left(T_{c j}\right)+\epsilon_{c j}>w_{c}^{2} \text {, and } v\left(T_{c k}\right)+\epsilon_{c k} \leq w_{c}^{2} .
$$

From Lemma 2 and the fact that $v$ is an increasing function we have $v\left(T_{c j}\right) \leq$ $\max \left\{v\left(T_{c i}\right), v\left(T_{c k}\right)\right\}$ for all $1 \leq i, j, k \leq m$. Thus, $v\left(T_{c j}\right) \leq v\left(T_{c i}\right)$ or $v\left(T_{c j}\right) \leq$ $v\left(T_{c k}\right)$. Noting that the two cases are similar, we only show that a contradiction 
ensues assuming the former inequality. If, instead the latter inequality holds, an analogous argument applies. This gives,

$$
-\frac{\epsilon}{2}<\epsilon_{c i} \leq w_{c}^{2}-v\left(T_{c i}\right) \leq w_{c}^{2}-v\left(T_{c j}\right)<\epsilon_{c j}<\frac{\epsilon}{2},
$$

where we have additionally used (12) and (15). This contradicts the assumption in the theorem that $\left|w_{c}^{2}-v\left(T_{c i}\right)\right|>\epsilon$.

Step 2. In this part of the proof we first fix two integers $1 \leq c, i \leq m$. With $r$ defined by Eq. (13) we can write $\vec{F}_{c}=\vec{V}_{r}+\vec{\eta}_{c}$. It follows directly from the condition of the theorem that $\vec{\eta}_{c}$ is of size $o(\sqrt{D})$. Thus,

$$
\begin{aligned}
\left|\vec{F}_{c}-\vec{V}_{i}\right|^{2} & =\left|\vec{V}_{r}-\vec{V}_{i}+\vec{\eta}_{c}\right|^{2} \\
& =\left|\vec{V}_{r}-\vec{V}_{i}\right|^{2}+2 \vec{\eta}_{c} \cdot\left(\vec{V}_{r}-\vec{V}_{i}\right)+\left|\vec{\eta}_{c}\right|^{2},
\end{aligned}
$$

so

$$
\begin{aligned}
\left|\frac{\left|\vec{F}_{c}-\vec{V}_{i}\right|^{2}}{D}-v\left(T_{r i}\right)\right| & \leq\left|\frac{\left|\vec{V}_{r}-\vec{V}_{i}\right|^{2}}{D}-v\left(T_{r i}\right)\right|+\left|\frac{2 \vec{\eta}_{c} \cdot\left(\vec{V}_{r}-\vec{V}_{i}\right)}{D}\right|+\left|\frac{\left|\vec{\eta}_{c}\right|^{2}}{D}\right|^{2} \\
& \leq\left|\frac{\left|\vec{V}_{r}-\vec{V}_{i}\right|^{2}}{D}-v\left(T_{r i}\right)\right|+2\left|\frac{\vec{\eta}_{c}}{\sqrt{D}}\right|\left|\frac{\vec{V}_{r}-\vec{V}_{i}}{\sqrt{D}}\right|+\left|\frac{\vec{\eta}_{c}}{\sqrt{D}}\right|^{2},
\end{aligned}
$$

where $\frac{\left|\vec{V}_{i}-\vec{V}_{j}\right|^{2}}{D}-v\left(T_{i j}\right) \stackrel{\mathrm{P}}{\rightarrow} 0$ as $D \rightarrow \infty$ by the second part of the proof of Theorem 1. Since $\left|\frac{\vec{V}_{r}-\vec{V}_{i}}{\sqrt{D}}\right| \stackrel{P}{\rightarrow} v\left(T_{r i}\right) \leq 1$ as $D \rightarrow \infty$ and $\left|\frac{\vec{\eta}_{c}}{\sqrt{D}}\right| \stackrel{\mathrm{P}}{\rightarrow} 0$ as $D \rightarrow \infty$, we have

$$
\frac{\left|\vec{F}_{c}-\vec{V}_{i}\right|^{2}}{D} \stackrel{\mathrm{P}}{\rightarrow} v\left(T_{r i}\right) \text { as } D \rightarrow \infty .
$$

The rest of the proof is now identical with the proof of Theorem 1, continuing from Eq. (10).

As we will show in the following, the condition on the distance between the foraging traits of consumers and the vulnerability traits of their central resources stated in the Theorem 2 is sharp in the sense that, when foraging traits have a distance of the order of $D^{1 / 2}$ or larger from the vulnerability traits of any resource item, then intervality is not guaranteed in the limit $D \rightarrow \infty$. On the other hand, it is not difficult to see that distances between vulnerability traits grow as $D^{1 / 2}$ as $D \rightarrow \infty$. The condition of theorem 2 therefore requires that foragers "single out" one particular resource item to which they are, up to small deviations, specialized. It is not clear if foragers typically have such a "preferred" resource in nature. One might therefore wonder if there are other constraints on foraging traits that also imply intervality, which could plausibly be satisfied in nature. One idea that comes to mind is that consumers will adapt their foraging traits so as to lie somewhere in the midst of the vulnerability traits of their resources. But, as the following theorem specifies, this alone is not be sufficient to ensure the COP. 
Theorem 3. With the same assumptions otherwise as in Theorem 2, if instead

$$
\frac{\left|\vec{F}_{c}-\vec{V}_{r_{D}}\right|^{2}}{D} \stackrel{\mathrm{P}}{\not} 0 \text { as } D \rightarrow \infty
$$

the food web does not necessarily have the consecutive ones property. This is true even if for all $q>1$,

$$
\frac{\left|\vec{F}_{c}-\vec{V}_{r_{D}}\right|^{2}}{D^{q}} \stackrel{\mathrm{P}}{\rightarrow} 0 \text { as } D \rightarrow \infty .
$$

In fact, it is possible to construct a food web where the foraging trait vectors of consumers are averages of their resources's vulnerability trait vectors, but which does not have the consecutive ones property.

Proof. See Appendix A.

Another conceivable constraint leading to food-web intervality may be that, while consumers are not focused or nearly focused on their preferred resources in every food web, they are focused on some conceivable resources species which may exist somewhere else, or only hypothetically. Relevant for this situation is the following lemma:

Lemma 4. Let $S=\left\{s_{1}, \ldots, s_{m}\right\}$ be a finite set of species. With each species $s_{i}$ we associate a trait vector $\left(\vec{V}_{i}, \vec{F}_{i}, w_{i}\right)$. Let $S^{\prime}$ be a subset of $S$. If the induced food web $L$ of $S$ has the COP, then the induced food web of $S^{\prime}$, given by $L^{\prime}=$ $\left\{(r, c): r \in S^{\prime}\right.$ and $\left.c \in S^{\prime}\right\}$ has the $C O P$.

Proof. The adjacent matrix $A\left(L^{\prime}\right)$ of $S^{\prime}$ can be obtained by deleting certain rows and columns in the adjacency matrix $A(L)$ of $S$. The COP is obviously conserved under the deletion of rows and under deletion of columns. It follows that $L^{\prime}$ has the COP, and so the corresponding food web.

Remark 6. Lemma 4 together with Theorem 1 imply that consumers need not necessarily be focused on their resources to guarantee intervality as $D \rightarrow \infty$. It suffices that consumers are focused on resource traits of "likely species", i.e., species in the community in question, in some other community (Rossberg, 2008), or species that could possibly exist, as long as its phylogenetic distance to all extant species is kept fixed as $D \rightarrow \infty$. This situation is not directly covered by Theorems 1 or 3, because, if such a potential species has positive phylogenetic distance to all extant species $1 \leq i \leq m$, then, $D^{-1}|\vec{F}-\vec{V}|^{2}$ will not converge to zero in probability as $D \rightarrow \infty$.

\section{Discussion}

In this paper, we have derived mathematically rigorous conditions for food-web intervality. These conditions encompass the key result obtained by Rossberg et al (2010a) through simulations, namely that food webs from a model with focused consumers and phylogenetic correlations tend to be interval in highdimensional niche spaces. However, as a result of our rigorous treatment, we were able to go far beyond the analysis of a single model, capturing instead 
a large model class that allows considerable variations in the way (1) how consumers (foraging traits) are distributed over their resources (vulnerability traits), (2) how niche widths vary between consumers, and (3) how phylogenetic trees are structured. The analysis also allowed us to determine condition for food-web intervality with weakly focused consumers in high-dimensional niche spaces.

In our analysis of food-web intervality, we assumed a high symmetry between the $D$ components of the vulnerability trait vectors. In reality, some traits will be more important for trophic interactions, thus contributing more to the trophic "distance" between species, others less, and there may be complicated interrelations. As has been argued elsewhere (Rossberg et al, 2010b), appropriate coordinate transformations in trait space might nevertheless allow us to derive a diagonal trophic distance measure of the form $\Delta\left(\vec{V}_{i}, \vec{F}_{j}\right)=$ $\sum_{l=1}^{d} \lambda_{l}\left(V_{i}^{(l)}-F_{j}^{(l)}\right)^{2}$, with the superscript $(l)$ denoting vector components, and it is not difficult to see that this transformation can be done such that the co-variance matrix of the components of $\vec{V}_{i}$ over a food web becomes the unit matrix. When the consumer $j$ is focused on a resource $r$, this corresponds to a distance between vulnerability traits $\Delta\left(V_{i}, V_{r}\right)$. Our results could be generalized to handle this case. Decisive for Theorem 1, for example, is the convergence in probability, as $D \rightarrow \infty$, of the (appropriately normalized) distance between vulnerability traits of two species to some increasing function of their phylogenetic distance. For any finite $D$, the degree to which intervality can be expected to be observed depends on the coefficient of variation $(\mathrm{CV})$ of trophic distance for fixed phylogenetic distance. In essence, the theorem requires this $\mathrm{CV}$ to go to zero. For the Euclidean distance measure this is the case when increasing $D \rightarrow \infty$, but other families $\Delta_{k}$ of distance measures will achieve the same as $k \rightarrow \infty$, as long as the CV at fixed phylogenetic distance goes to zero. When vulnerability traits derive from branching Ornstein-Uhlenbeck processes of the form considered here, the $\mathrm{CV}$ of the diagonal distance measure defined above evaluates to $\mathrm{CV}=\left(2 \sum_{l} \lambda_{l}^{2}\right)^{1 / 2} / \sum_{l} \lambda_{l}$. For Euclidean distances this becomes $\mathrm{CV}_{D}=(2 / D)^{1 / 2}$. By equating these values, we can define an effective dimension $D_{\text {eff }}=2 / \mathrm{CV}^{2}=\left(\sum_{l} \lambda_{l}\right)^{2} / \sum_{l} \lambda_{l}^{2}$. Large $D_{\text {eff }}$ generally indicate that pronounced intervality must be expected by the phylogenetic mechanism.

There is a simple intuitive explanation for why food webs are interval in high-dimensional trophic niche spaces. First, the resources covered by the diet of a consumer tend to be similar. As species that share a common ancestor in the recent past tend to be similar, the diet of a consumers will often encompass an entire clade (branch) of the phylogenetic tree. Second, with high-dimensional trait vectors, the chance that two species that did not descend from a common ancestor in the recent past are similar is very small, as this would require most components of their vulnerability trait vectors to match. This means that a consumer will typically include an entire clade of the phylogenetic tree in its diet, and no other species. Hence, the food web has the consecutive ones property with the ordering induced by the phylogenetic tree. Stated differently, repeated branching of the mean-reverting Ornstein-Uhlenbeck process builds up phylogenetic correlations in vulnerability trait vectors. With increased dimensionality, the time since separation of two lineages becomes more informative about the actual ecological differences, leading to stronger phylogenetic correlations and consequently interval food webs. 
The two major ecological assumptions that underlie our results are fast evolution of foraging traits relative to vulnerability traits and 'focusedness', i.e. foraging traits of consumers that are located at or near the vulnerability traits of specific resources. The assumption of fast evolution of foraging traits is supported by empirical observations indicating that phylogenetic correlations among foraging traits are weak (Cooper and Vitt, 2002; Blomberg et al, 2003b; Bersier and Kehrli, 2008). In particular, taxonomically related resources tend to be preyed upon by similar consumers, while taxonomically related consumers to a lesser extend tend to prey on the same resources. This is the expected pattern when foraging traits evolve faster than vulnerability traits. The conclusion is further reinforced by a quantitative study by Rossberg et al (2006a) in which a food-web model was fitted to empirical data, allowing the inference of the relative rates of evolutionary change in the two trait vectors. We thus believe that it is reasonable to assume time-scale separation between the evolution of vulnerability and foraging traits.

While fast consumer adaptation is supported empirically, there is no corresponding empirical support for the assumption that a consumer's foraging traits are focused on a focal resource's vulnerability traits. In Sect. 4 we partly relaxed this assumption by instead assuming that a similar condition holds asymptotically as the dimension $D \rightarrow \infty$. We also considered two alternative rules for the placement of a consumer's foraging traits: at the position in niche space given by the average of its resources vulnerability traits or focused on vulnerability traits of a resource which may exist somewhere else, or only hypothetically. Interestingly, the former does not lead to intervality while the latter does. In Rossberg et al (2010a) we numerically explored a third rule by allowing foraging traits to evolve in the direction of increased fitness according to an $a$ priori defined fitness measure. Simulated food-webs then tended to be interval in high-dimensional trait-spaces, too. Yet, a re-analysis of the data (not shown) later revealed that the squared distances between foraging traits and the nearest vulnerability traits were of the same order as the number of dimensions. The observed intervality at high dimensions in these simulations is hence not covered by the analytic results derived here. Further work will therefore be needed to understand the full extent to which our analytic results can be generalized to non-focused consumers. On the other hand, one needs to keep in mind that intervality in empirical food webs is clearly not perfect. Even the model by tested by Rossberg et al (2006a), which does not invoke consumer adaptation at all and relies on a much simpler representation of trophic niche space than used here, still overestimates empirical intervality more often than underestimating it.

Another step towards increased ecological realism in food-web models is to explicitly simulate the population dynamics of all species in evolving food webs (e.g., Post and Pimm, 1983; Caldarelli et al, 1998). Unfortunately, intervality is not generally studied in such models. An exception may be the Population Dynamical Matching Model (Rossberg et al, 2008, Tab. 2), for which $D_{\text {diet }}$, a measure for how well the COP is satisfied (Cattin et al, 2004), has been evaluated. Simulation values for $D_{\text {diet }}$ were in the range $0.33 \pm 0.20$ (mean $\pm \mathrm{SD}$ ), overlapping but slightly larger than the empirical range $0.22 \pm 0.15$. The reason for under-develop intervality in these simulations might be that the niche-space dimensionality of $D=5$ used in the model was still comparatively low. 
In order to better understand the phenomenon of consumer-resource coevolution and its effects on intervality, the evolution of food webs could be studied using, for example, techniques of adaptive dynamics (Dieckmann and Law, 1996; Geritz et al, 1998). In the adaptive dynamics framework, invasion fitness is derived from the underlying model, removing the need for an externally imposed fitness measure or ad hoc assumptions such as that of focused consumers. This objective appears to be within reach numerically, but, because of the intricate feed-back loops involved, will be very hard to incorporate into a rigorous mathematical theory. A more promising avenue from a mathematical perspective would to move beyond intervality and to study other aspects of food-web structure, such as degree distributions or the block structure of adjacency matrices (Critchlow and Stearns, 1982) expected to accompany intervality in high dimensions.

By developing a mathematical theory for a class of models that encompasses the one proposed by Rossberg et al (2010a), this paper adds to a growing suite of food-web models which are well-understood analytically, such as the cascade model (Cohen et al, 1990), the niche model (Camacho et al, 2002; Allesina et al, 2008), the speciation model (Rossberg et al, 2006b), or the matching model (Rossberg, 2008). With a large number of analytically tractable foodweb models to work with, we expect that the mechanisms behind the emergence of more aspects of food-web structure will be pinned down in the years to come.

\section{Acknowledgements}

This research has been supported by the European Marie Curie Research Training Network FishACE (Fisheries-induced Adaptive Changes in Exploited Stocks), funded through the European Community's Sixth Framework Programme (Contract MRTN-CT-2004-005578). A.G.R. is supported by a Beaufort Marine Research Award, carried out under the Sea Change Strategy and the Strategy for Science Technology and Innovation (2006-2013), with the support of the Marine Institute, funded under the Marine Research Sub-Programme of the Irish National Development Plan 2007-2013. We thank Ulf Dieckmann and Klas Markström for useful discussions.

\section{A Proof of Theorem 3}

Before proving Theorem 3, we derive a useful formula for expectation values of quadratic expressions in vulnerability traits. Denote the $i$ th component of a vector $\vec{V}$ by $V^{(i)}(1 \leq i \leq D)$. Choose an arbitrary species $m$ as a reference point. From the bi-linearity of covariances and recalling that $\operatorname{var}(x)=\operatorname{cov}(x, x)$, one gets

$$
\begin{aligned}
\operatorname{var}\left(V_{k}^{(i)}-V_{l}^{(i)}\right) & =\operatorname{var}\left[\left(V_{k}^{(i)}-V_{m}^{(i)}\right)-\left(V_{l}^{(i)}-V_{m}^{(i)}\right)\right] \\
& =\operatorname{var}\left(V_{k}^{(i)}-V_{m}^{(i)}\right)-2 \operatorname{cov}\left(V_{k}^{(i)}-V_{m}^{(i)}, V_{l}^{(i)}-V_{m}^{(i)}\right)+\operatorname{var}\left(V_{l}^{(i)}-V_{m}^{(i)}\right) .
\end{aligned}
$$

Using Lemma 3, we can now express variances in terms of the function $v$ 
defined by Eq. (5). Solving for the remaining covariance gives

$$
\operatorname{cov}\left(V_{k}^{(i)}-V_{m}^{(i)}, V_{l}^{(i)}-V_{m}^{(i)}\right)=\frac{v\left(T_{k m}\right)+v\left(T_{l m}\right)-v\left(T_{l m}\right)}{2} .
$$

Because all vulnerability traits derive from the same ancestor in a random walk, they all have the same expectation value. Thus

$$
E\left[\left(V_{k}^{(i)}-V_{m}^{(i)}\right)\left(V_{l}^{(i)}-V_{m}^{(i)}\right)\right]=\frac{v\left(T_{k m}\right)+v\left(T_{l m}\right)-v\left(T_{l m}\right)}{2} .
$$

We are now able to prove Theorem 3. Note that the proof constructs a food web where the foraging trait vectors of all consumers are located at the center of mass of the vulnerability trait vectors of their resources, but which is nonetheless not interval.

Theorem 3. We construct a minimal three-species food web such that as $D \rightarrow \infty$ it will have the following adjacency matrix with probability 1 :

$$
A(L)=\left(\begin{array}{lll}
0 & 1 & 1 \\
1 & 0 & 1 \\
1 & 1 & 0
\end{array}\right)
$$

By inspecting all 6 possible permutations of rows, it is clear that this adjacency matrix does not have the COP. We assume a phylogenetic tree of three species as shown schematically in Fig. 1 a). That is, we assume that the lineage of species 1 branched off before the lineages of species 2 and 3 separated. With such a phylogenetic tree, $v\left(T_{12}\right)=v\left(T_{13}\right)$. Further, let the foraging traits be given by the centers of mass between two vulnerability traits, i.e. $\vec{F}_{1}=\left(\vec{V}_{2}+\vec{V}_{3}\right) / 2$, $\vec{F}_{2}=\left(\vec{V}_{1}+\vec{V}_{3}\right) / 2$ and $\vec{F}_{3}=\left(\vec{V}_{1}+\vec{V}_{2}\right) / 2$. Then, as $D \rightarrow \infty$, we get using Eq. (16):

$$
\frac{\left|\vec{F}_{2}-\vec{V}_{1}\right|^{2}}{D}=\frac{\left|\vec{F}_{2}-\vec{V}_{3}\right|^{2}}{D} \stackrel{\mathrm{P}}{\rightarrow} \frac{v\left(T_{13}\right)}{4}
$$

and

$$
\frac{\left|\vec{F}_{2}-\vec{V}_{2}\right|^{2}}{D} \stackrel{\mathrm{P}}{\rightarrow} \frac{1}{4} v\left(T_{12}\right)+\frac{1}{2} v\left(T_{23}\right)>\frac{v\left(T_{12}\right)+v\left(T_{23}\right)}{4} .
$$

By symmetry we have

$$
\frac{\left|\vec{F}_{3}-\vec{V}_{1}\right|^{2}}{D}=\frac{\left|\vec{F}_{3},-\vec{V}_{2}\right|^{2}}{D} \stackrel{\mathrm{P}}{\rightarrow} \frac{v\left(T_{12}\right)}{4}
$$

and

$$
\frac{\left|\vec{F}_{3}-\vec{V}_{3}\right|^{2}}{D} \stackrel{\mathrm{P}}{\rightarrow} \frac{1}{4} v\left(T_{13}\right)+\frac{1}{2} v\left(T_{23}\right)>\frac{v\left(T_{13}\right)+v\left(T_{23}\right)}{4} .
$$

For the remaining consumer,

$$
\frac{\left|\vec{F}_{1}-\vec{V}_{2}\right|^{2}}{D}=\frac{\left|\vec{F}_{1}-\vec{V}_{3}\right|^{2}}{D} \stackrel{\mathrm{P}}{\rightarrow} \frac{v\left(T_{12}\right)}{4},
$$


and

$$
\frac{\left|\vec{F}_{1}-\vec{V}_{1}\right|^{2}}{D} \stackrel{\mathrm{P}}{\rightarrow} v\left(T_{12}\right)-\frac{v\left(T_{23}\right)}{4}>\frac{v\left(T_{12}\right)}{2} .
$$

We now choose the niche width $w_{2}=\sqrt{\frac{1}{4}\left(v\left(T_{12}\right)+v\left(T_{23}\right)\right)}, w_{3}=\sqrt{\frac{1}{4}\left(v\left(T_{13}\right)+v\left(T_{23}\right)\right)}$ and $w_{1}=\sqrt{\frac{1}{2} v\left(T_{12}\right)}$. The induced food web

$$
L=\left\{(r, c):\left|\vec{V}_{r}-\vec{F}_{c}\right|^{2}<D w_{c}^{2}: c=1,2,3 \text { and } r=1,2,3\right\}
$$

is equivalent to the above adjacency matrix $A(L)$, which does not have the COP. Finally, since

$$
\frac{\left|\vec{F}_{c}-\vec{V}_{r_{D}}\right|^{2}}{D^{q}}
$$

converges in probability to a finite value for $q=1$ as $D \rightarrow \infty$, it follows that the limit is 0 in probability for $q>1$.

\section{References}

Allesina S, Pascual M (2009) Food web models: A plea for groups. Ecology Letters 12:652-662

Allesina S, Alonso D, Pascual M (2008) A general model for food web structure. Science 320:658-661

Benzer S (1959) On the topology of genetic fine structure. Proceedings of the National Academy of Sciences of the USA pp 1607-1620

Bersier LF (2007) A history of the study of ecological networks. In: Képès F (ed) Biological Networks, World Scientific, New Jersey, chap 11, pp 365-421

Bersier LF, Kehrli P (2008) The signature of phylogenetic constraints on foodweb structure. Ecological Complexity 5(2):132-139

Blomberg SP, Garland T, Ives AR (2003a) Testing for phylogenetic signal in comparative data: behavioral traits are more labile. Evolution 57:717-745

Blomberg SP, Garland T, Ives AR (2003b) Testing for phylogenetic signal in comparative data: behavioral traits are more labile. Evolution; International Journal of Organic Evolution 57(4):717-745

Caldarelli G, Higgs PG, McKane AJ (1998) Modelling coevolution in multispecies communities. J Theor Biol 193:345

Camacho J, Guimerà R, Amaral LAN (2002) Analytical solution of a model for complex food webs. Phys Rev E 65:030,901R

Cattin MF, Bersier LF, Banasek-Richter C, Baltensperger R, Gabriel JP (2004) Phylogenetic constraints and adaptation explain food-web structure. Nature $427: 835-839$ 
Cohen JE (1977) Food webs and the dimensionality of trophic niche space. Proceedings of the National Academy of Sciences of the USA 74:4533-4536

Cohen JE (1978) Food Webs and Niche Space. Princeton Univeristy Press, Prinecton, NJ

Cohen JE, Briand F, Newman CM (1990) Community Food Webs: Data and Theory. Springer, Berlin

Cooper WE, Vitt LJ (2002) Distribution, extent, and evolution of plant consumption by lizards. Journal of Zoology 257(04):487-517

Critchlow RE, Stearns SC (1982) The structure of food webs. Am Nat 120(4):478-499

Dieckmann U, Law R (1996) The dynamical theory of coevolution: a derivation from stochastic ecological processes. Journal of Mathematical Biology 34:579612

Felsenstein J (1988) Phylogenies and quantitative characters. Annu Rev Ecol Syst 19:445-471

Gardiner C (2009) Stochastic Methods: a Handbook for the Natural and Social Sciences, 4th edn. Springer Verlag, Berlin Heidelberg

Geritz SAH, Kisdi E, Meszéna G, Metz JAJ (1998) Evolutionary singular strategies and the adaptive growth and branching of the evolutionary tree. Evolutionary Ecology 12:35-57

Grimmett GR, Stirzaker DR (1992) Probability and random processes, 2nd edn. The Clarendon Press Oxford University Press, New York

Millennium Ecosystem Assessment (2005) Ecosystems and Human Well-being: Synthesis. Island Press, Washington, DC

Mouillot D, Krasnov BR, Poulin R (2008) High intervality explained by phylogenetic constraints in host-parasite webs. Ecology 89(7):2043-2051

Pimm SL (1982) Food Webs. Chapman and Hall, New York

Post WM, Pimm SL (1983) Community assembly and food web stability. Math Biosci 64:169-192

Rossberg A, Brännström A, Dieckmann U (2010a) Food-web structure in lowand high-dimensional trophic niche spaces. Proceedings of the Royal Society Interface

Rossberg AG (2008) Part-whole relations between food webs and the validity of local food-web descriptions. Ecological Complexity 5(2):121-131,

Rossberg AG, Matsuda H, Amemiya T, Itoh K (2006a) Food webs: experts consuming families of experts. Journal of Theoretical Biology 241(3):552-563

Rossberg AG, Matsuda H, Amemiya T, Itoh K (2006b) Some properties of the speciation model for food-web structure - Mechanisms for degree distributions and intervality. Journal of Theoretical Biolpgu 238(2):401-415, 
Rossberg AG, Ishii R, Amemiya T, Itoh K (2008) The top-down mechanism for body-mass-abundance scaling. Ecology 89(2):567-580,

Rossberg AG, Brännström Å, Dieckmann U (2010b) How trophic interaction strength depends on traits - A conceptual framework for representing multidimensional trophic niche spaces. Theor Ecol 3(1):13-24

Semple C, Steel M (2003) Phylogenetics. Oxford University Press, USA

Stouffer DB, Camacho J, Amaral LAN (2006) A robust measure of food web intervality. Proc Natl Acad Sci USA 103(50):19,015-19,020

Sugihara G (1984) Graph theory, homology and food webs. In: Levin SA (ed) Lecture Notes in Biomathematics, American Mathematical Society, pp 83-101

Williams RJ, Martinez ND (2000) Simple rules yield complex food webs. Nature 404:180-183 